\title{
Correction to: Epidemiology of diabetes and diabetic complications in China
}

\author{
Ronald C. W. Ma ${ }^{1,2,3,4,5}$
}

Published online: 18 April 2018

(C) Springer-Verlag GmbH Germany, part of Springer Nature 2018

\section{Correction to: Diabetologia}

https://doi.org/10.1007/s00125-018-4557-7

Unfortunately, the reference given in the legend to Fig. 2 was incorrect. The legend should have stated that Fig. 2 was drawn based on data from reference [49].

\section{Reference}

49. Bragg F, Holmes MV, Iona A et al (2017) Association between diabetes and cause-specific mortality in rural and urban areas of China. JAMA 317:280-289

The online version of the original article can be found at https://doi.org/ $10.1007 / \mathrm{s} 00125-018-4557-7$

Ronald C. W. Ma

rcwma@cuhk.edu.hk

1 Department of Medicine and Therapeutics, The Chinese University of Hong Kong, Prince of Wales Hospital, 30-32 Ngan Shing Street, Shatin, New Territories, Hong Kong, Hong Kong Special Administrative Region, People's Republic of China

2 Hong Kong Institute of Diabetes and Obesity, The Chinese University of Hong Kong, Hong Kong Special Administrative Region, People's Republic of China

3 Li Ka Shing Institute of Health Sciences, The Chinese University of Hong Kong, Hong Kong Special Administrative Region, People's Republic of China

4 Chinese University of Hong Kong and Shanghai Jiao Tong University (CUHK-SJTU) Joint Research Centre in Diabetes Genomics and Precision Medicine, Hong Kong Institute of Diabetes and Obesity, The Chinese University of Hong Kong, Hong Kong Special Administrative Region, People's Republic of China

5 NHMRC Clinical Trials Centre, Sydney Medical School, The University of Sydney, Sydney, NSW, Australia 